\title{
A Quantum Computer in a "Chinese Room”
}

\author{
Vasil Penchev, vasildinev@gmail.com \\ Bulgarian Academy of Sciences: Institute of Philosophy and Sociology: \\ Dept. of Logical Systems and Models
}

\begin{abstract}
Pattern recognition is represented as the limit, to which an infinite Turing process converges. A Turing machine, in which the bits are substituted with qubits, is introduced. That quantum Turing machine can recognize two complementary patterns in any data. That ability of universal pattern recognition is interpreted as an intellect featuring any quantum computer. The property is valid only within a quantum computer: To utilize it, the observer should be sited inside it. Being outside it, the observer would obtain quite different result depending on the degree of the entanglement of the quantum computer and observer. All extraordinary properties of a quantum computer are due to involving a converging infinite computational process contenting necessarily both a continuous advancing calculation and a leap to the limit. Three types of quantum computation can be distinguished according to whether the series is a finite one, an infinite rational or irrational number.
\end{abstract}

Keywords: infinite computation, quantum computer, quantum Turing machine, quantum entanglement, qubit, universal pattern recognition

The article considers quantum computation both philosophically and mathematically only theoretically raising the following questions:

How can one reduce pattern recognition in a single mathematical model if the data are a numerical series?

Can that mathematical model be equivalently represented as a computation of a quantum computer?

The answers of these questions will be deduced successively:

\section{How Can One Reduce Pattern Recognition in a Single Mathematical Model if the Data Are a Numerical Series?}

Any data can be encoded as a numerical series, and this is the way for them to be represented in a computer. Indeed the most general model of any computer, with which one works, is a Turing machine [8], and its data are a Turing tape, i.e. a numerical series usually binary. Consequently the recognition of a pattern in a numerical series is a general enough task representative for pattern recognition at all.

Of course all data, which a standard computer can process, are always a finite set. However it generates new data in course of processing, which are usually interpreted as intermediate results, and the ultimate result can be yielded only when the Turing machine stops and it is or is contained in that ultimate state of it when it has just stop. If it does not stop a long enough time, maybe the algorithm of processing is wrong and it "cycles" ("hangs up", came to a standstill) granting that the 
result should finite. Of course if the problem is such as to calculate e.g. " $\sqrt{2}$ ", the result is not finite, and a good algorithm will never stop by itself, too.

One should distinct two cases: (1) the computer reaches the result, either correct or wrong, in a finite time; (2) it cannot reach the result in a given time, either for a wrong algorithm or for an infinite result or for it will reach the result in a time long than the given (the "halting problem"): One needs an exact criterion to distinct an infinite result from the absence of any result. Thus this is not the "halting problem" yet. One can approach as follows:

The Turing tape can be always interpreted as a binary fraction and thus as a real number either (1) rational or (2) irrational.

1. One obtains a finite set representing a fraction either as the set of digits until to the last "one” (all are zeros after it) or the sets of digits of the period of that fraction. In both of these subcases a finite pattern is recognized.

2. The binary fraction is infinite and no finite pattern can be recognized. Nevertheless the series of any $\mathrm{n}$ first digits is converging to some irrational number where $\mathrm{n}$ converges to infinity. Just this irrational number can be accepted as the pattern recognizable unambiguously. The criterion of convergence comprises the former case, too. Consequently that convergence is a general equivalent to the existence of any result either finite or infinite.

Furthermore one needs to consider the more general case where the Turing machine continues to work for an indefinitely long time raising just the "halting problem" and producing new Turing tapes again and again. Now the questions is what is necessary and sufficient condition for the Turing machine to obtain a result working for either a finite or an infinite time. Meaning the above criterion of convergence, the sufficient condition is: it should just go ahead neither repeating the processing of one and the same cell nor going back to process the previous cell. This is equivalent to a well-ordering in the work of the Turing machine and thus to the axiom of choice as to the set of states (and thus of "tapes") of the Turing machine. The necessary condition is the above sufficient condition to happen for a finite time after the Turing machine has started working.

Immediately one should reformulate the "halting problem" thus: What is searched is an equivalent transformation (or otherwise said invariance) of an arbitrary Turing machine moving back and forth into another moving only forth. If that transformation exists, the Turing machine will not cycle though it can run indefinitely. Moreover if one constructs a suitable topology to guarantee always that invariance, any computation represented by mappings of neighborhoods analogical to the work of a Turing machine will have an exact result. That topology will be equivalent to universal pattern recognition.

Indeed the pattern recognition in all cases enumerated above means to reveal a limit, to which the series of Turing tapes obtained successively in course of the work of the machine converge:

If it has stopped for a finite time, that limit coincides with its last state, which is the ultimate result of its work. No leap to reach the limit is necessary since the limit is or more exactly coincides with an element (the last one) of the series.

However if it cannot stop in any finite time and the limit exists, which is equivalent to the above necessary and sufficient condition for it, the limit and thus the pattern recognized needs a finite jump to be reached for any finite time after the machine has started working. The subcases are correspondingly two: The limit is a rational (3) or irrational (4) number:

3. Though the limit needs some finite leap beyond any result of the work of the Turing machine for any finite time, there is a one-to-one correspondence of the limit and a repeating sequence of digits occurring constantly in course of working, namely the period of the fraction. In that sense one can say that the limit is contented in a long enough period of time, during which the machine has been working. However no one can be sure that the revealed "inductively" pattern will indeed repeat always further. 
4. The limit needs unconditionally some jump to be perceived. This case is intuitively the boundary between an automaton, computer, Turing machine, etcetera, on one hand, and an intellect, including that of a human being, on the other hand. Consequently if one demonstrates that some device can do those leaps in any case when they are necessary, that device will deserve to be called an artificial intellect. The answer of the second question raised in the beginning will display just this, namely a quantum computer possesses that property however only intrinsically or immanently in a sense.

Before continuing, the obtained result will be translated from the "Turing language" into that of partial and recursive functions, which is known to be equivalent, but nevertheless it offers a different interpretation and thus viewpoint to computation. The leap from any member of a converging series to its limit in a Turing machine is equivalent to some minimization operator transforming a primitive recursive function of a parameter to the same function with the zero value of that parameter. How that value will be reached for an indefinite, perhaps even infinite number of recursions, and thus it is represented just by a "WHILE" operator: "DO something (according to the primitive recursive function) WHILE $p$ (the parameter) $=0$ ". Furthermore any recursive function can replace the primitive one. Then one can define any jump and thus 'the understanding of an artificial intellect' by a suitable total non-primitive recursive function guaranteeing this leap for any values of the input variables.

If the $\lambda$-calculus is utilized to represent such a jump instead of the apparatus of recursive functions or that of Turing machines, this would add nothing new in the interpretation. Indeed the $\lambda$ calculus is an intermediate link between those two, transforming the Turing tape into an anonymous function with a single variable in the way to the named recursive functions. Consequently the leap will be represented by the initial and by ultimate state both specified by the parameter involved before that in course of calculation.

The common in the three representation enumerated above is a "quantum" understanding of 'intellect' as a leap, to which one juxtaposes a continuous trajectory symbolized by the successive cells of the Turing tape or by the corresponding recursions or $\lambda$-calculations between the initial and the ultimate state of the leap. The number of those cells or recursions, or $\lambda$-calculations is indefinite and even infinite. This correspondence is the way for the leap of 'understanding' as an insight suddenly revealing truth to be formally represented in the well-developed theoretical language of calculus. As one can see a little further, that formalization of any act of an intellect and thus of an intellect in general obeys the objectivity it to be realized as a quantum computer.

Summarizing the answer of the first question, one can say that pattern recognition can be universally represented as the limit, to which the series of successive partial calculations converges. The necessary and sufficient condition for that limit to exist is a well-ordering of these successive partial calculations requiring the axiom of choice to be always guaranteed. In turn that general formal model of pattern recognition allows of defining mathematically any intellectual act of understanding as a jump from some finite set of data over a gap of indefiniteness and maybe even infinity to the limit to which the series of those data converges since it is the pattern recognizable in the data. Furthermore that model can distinguish three cases from each other:

The first case is that of a 'zero leap' where the completed calculation is finite and the limit as well as the recognized pattern is the ultimate calculative state. Any standard computer can "understand" in this way, but that "understanding” being corresponding to the leap is also zero.

The second case can be called inductive being intermediate between the "understanding" in the first one and the understanding in a proper sense in the third one: The recognizable pattern appears constantly and in equal periods of partial calculations. However a leap needs yet for the calculation to stop and the obtained partial result to be confirmed as the ultimate one. For example, the criterion for a standard computer to stop should be formulated as any, even maybe incredibly complex recursive function, but which must be a primitive one, and thus its "understanding" is again zero. How- 
ever if one decides that the number of inductive repetitions is "enough" ipso facto making a jump without any exact criterion in the form a primitive recursive function, that understanding can be designated as inductive and it is yet inaccessible to a contemporary computer.

The third case requires some nonzero leap unconditionally for the limit, to which a necessarily infinite series of partial calculations converges, does not coincide with any subset of partial calculations. That understanding corresponding to the nonzero leap is a one in a proper sense. It is granted as the sufficient condition of intellect. It is not a necessary one since any intellect, e.g. a human being, can accomplish finite calculations with a "zero leap" or "zero understanding” like a standard computer.

\section{Can That Mathematical Model Be Equivalently Represented as a Computation of a Quantum Computer?}

The meant model can be considered both as that of any pattern recognition and thus furthermore as that of an intellect.

Quantum computer can be introduced in a few ways: as a quantum Turing machine [3] or as quantum circuits [5], for which [9] has proved to be equivalent. Besides one can utilize a simple generalization of a Turing machine where all cell are replaced by quantum ones or otherwise said, any bit with a quantum bit (qubit) as follows:

Any cell of a Turing tape is a bit where a bit is the elementary unit of information, the choice between two equiprobable alternatives usually designated as " 0 " or " 1 ". A qubit, which replace a bit, is defined so:

$$
\alpha|0\rangle+\beta|1\rangle=1 \text { qubit }
$$

“ $\alpha, \beta$ ” are two complex numbers such that “ $|\alpha|^{2}+|\beta|^{2}=1$ ”, and “ $|0\rangle,|1\rangle$ ” are two orthogonal subspaces in Hilbert space or correspondingly, their bases.

The empty cell of a qubit can be represented as a unit ball in the usual 3D Euclidean space, in which one can choose two points representing the value assigned to that qubit: the one can be any point of the ball, but the other should be only in its surface (a unit sphere) obeying " $|\alpha|^{2}+|\beta|^{2}=$ 1 ”. The two orthogonal subspaces “ $|0\rangle,|1\rangle$ ” are represented as any two orthogonal great circles of the ball.

Consequently if a standard bit is a choice between two alternatives and can be thought as a finite choice, a qubit is a choice from a continuum of equiprobable alternatives and requires in general the axiom of choice in that sense to be able to be always made. However if one knows the values of " $\alpha$ " and " $\beta$ ", the axiom of choice is not necessary for the corresponding point to be chosen: " $\alpha$ " and " $\beta$ " are the values of that qubit. The choice once made and displaying those values can be always repeated already without needing the axiom of choice.

Since any two successive "axes" of Hilbert space such as $e^{i n \omega}, e^{i(n+1) \omega}$ represent two orthogonal subspaces of it, any point (vector) in Hilbert space has a qubit equivalent as follows:

Given any vector $\vec{V}$ of the complex Hilbert space with components $C_{1}, C_{2}, \ldots, C_{n}, C_{n+1}, \ldots$ in "axes”, $1^{\text {st }}, 2^{\text {nd }}, \ldots, n^{\text {th }}, n+1^{\text {th }}, \ldots$, one can construct a one-to-one mapping of it into its representation in qubits, e.g. so:

If both consecutive components, $C_{n}=0$ and $C_{n}=0: \alpha_{n}=0, \beta_{n}=0$ (conventionally).

If the consecutive components " $C_{n} \neq 0$ " or " $C_{n+1} \neq 0$ ”:

$$
\alpha_{n}=\frac{C_{n}}{\sqrt{\left|C_{n}\right|^{2}+\left|C_{n+1}\right|^{2}}}, \beta_{n}=\frac{C_{n+1}}{\sqrt{\left|C_{n}\right|^{2}+\left|C_{n+1}\right|^{2}}}
$$

Consequently any point in Hilbert space interpretable as a "wave function" in quantum mechanics and thus as a state of a quantum system is a quantum Turing tape consisting of well-ordered 
qubits, and any observable definable as a self-adjoint operator is an elementary Turing computation transforming a wave function into another in such a way that the serial number of any qubit is kept: One can easily see that the conservation of the serial number in a quantum computation is equivalent to that: the corresponding operator to be just self-adjoint.

Furthermore one can introduce a special regime for a Turing machine to run: where all cells are processed in parallel so that the ultimate state comprising even an infinite number of successive cells of the Turing tape in general can be reached in a single working cycle, i.e. by a jump equivalent both to the universal recognition of an intellect and to resolving the halting problem. However any Turing machine in the standard regime of successive processing, i.e. cell by cell, will differ from the former by an infinite number of filled cells even if they content only zeros.

One can demonstrate that both regimes would coincide as to a quantum Turing machine as that described above processing qubits instead of bits. The same property is also known as the principle of quantum parallelism usually granted. As to the pattern recognition (formalized as above) of a quantum computer, this means that it recognizes always a pattern as an intellect, but in a sense restricted in the framework of quantum complementarity, which will be carefully elucidated further.

One can justify that the parallel and serial processing of a quantum computer coincide as follows: The parallel processing means to be made an infinite set of choices (the Turing tapes of qubits), each of which in turn chooses an element among infinitely many (all values of a single qubit). In final analysis this is equivalent to being made a choice among infinitely many, i.e. all processing of a quantum Turing machine is reducible to the equivalent processing of a single qubit for the infinity of infinities is not more than one infinity by dint of the axiom of choice in general, which is not necessary in the case of question. And the serial processing means some finite set of qubits to be treated, which is equivalent to working up the infinitely many qubits of a Turing tape in parallel for that finite set of qubits is just as infinite as an infinite set of ones or as a single qubit. The difference of a quantum Turing machine from a standard one is due to the fact that the serial processing of any finite sets of bits being finite choices is never equivalent to the parallel processing of an infinite set of bits. Thus the parallel and serial processing of a quantum computer coincide always while those of a standard one can coincide only as to a finite set of bits being due to the different properties correspondingly of infinity or finiteness.

The same intriguing merit of a quantum computer can be demonstrated in pattern recognition represented as the leap to the limit of any converging series. Even more (though it is "less" in a sense), the quantum computer recognizes (or "imagines" in the same sense) a pattern in any infinite series (i.e. including the case where the series is not converging). One can say that the ability of imagination as it will be formalized a little below is a necessary quality of any intellect including that of a quantum computer.

One can consider two ways the limit of a converging series to be reached:

- Instantly, by jump, and as to the pattern recognition, by “insight” or by parallel processing, and:

- Successively, in a continuous trajectory, and as to the pattern recognition, by "persistence" or by serial processing.

However since one and the same limit is reached in both ways, they can be equated in a sense, which is interpreted in quantum mechanics as "wave-particle duality” e.g. meaning the equivalence of all trajectories both smooth and jump-like between two points in the Feynman interpretation. That equating can be symbolized so:

$$
\{a, b, d\} \leftrightarrow\{a, b, d(t)\}: \Psi(x)
$$

Here $a, b$ mean in fact anything as long as some distance $d$ can be defined between them, and $d(t)$ is a normal (i.e. non-generalized) function of some parameter t interpretable as time for the "normality" of the function. Rather unexpectedly, that always defines a "wave function" $\boldsymbol{\Psi}(x)$ de- 
fined over all space of " $x$ ", where the distance $d$ between the elements of the set, to which $a$ and $b$ belong, has been introduced. In fact that introduction of 'wave function' explicates the equivalence of a single qubit (in the left side) and a wave function discussed above.

Furthermore $\boldsymbol{\Psi}(x)$ implies a Hilbert space, being a basis in it. Being anything, " $a, b$ " can be interpreted as two states of a quantum system as the beginning and end of a computation representable as the first element and limit of a converging series. Besides the distance "d" can be also interpreted as a function of time, but this function should be a generalized, "jump-like" one:

$$
d_{0}(t)=\left\{\begin{array}{c}
t \in(-\infty, 0): d(t)=0 \\
t=0: \dot{d}=\delta(0), \text { the Dirac } \delta \text { function } \\
t \in(0,+\infty): d(t)=d
\end{array}\right.
$$

Meaning that, the "insight" of a jump to the limit and the recursive construction of a series converging to the limit are correspondingly represented by a generalized function " $d_{0}(t)$ " and a normal (non-generalized) but continuous (but not smooth) function " $d(t)$ ", and the values " $d_{0}$ " of the former and those of the latter " $d$ " coincides: This means that the big leap from 0 to $d$ is decomposed to little jumps corresponding to the each successive transition from an element of the series to the next.

Furthermore if the jump $d_{0}(0)$ of $d_{0}(t)$ is given, the progress in time of $d(t)$ to be constructed requires the axiom of choice. The development of an "instant", that of the jump " $d_{0}(0)$ ", into the well-ordered temporal sequence of the non-generalized function $d(t)$ needs it in general. However this implies rather extraordinary corollaries as it is usual once the axiom of choice is involved:

If the series is not converging, the axiom of choice always allows of it to be monotonically reordered, and if it is restricted in any finite interval, it is necessarily converging. The normalization by qubit representation as above guarantees that the series is always restricted in some finite interval and thus any series can be reordered in a way to be converging: In the present context, this means that any data can be reordered in a way a pattern to be recognizable from them. However can the initial and the reordered data to be granted as equivalent? That would mean an invariance of the data to reordering, rather ridiculous at first glance, and thus to the axiom of choice in general, too.

Nevertheless, the theorems of the absence of hidden variables [6-7] in quantum mechanics guarantee just this: Indeed, the state of a quantum system before measurement is fundamentally unorderable for them, e.g. as $d_{0}(0)$ is such, and the same after measurement is well-ordered in a series of results, e.g. as $d(t)$ is such. As one can convince above, the mapping $d_{0}(0) \rightarrow d(t)$ requires the axiom of choice, and that $d_{0}(0)$ itself excludes it: Consequently $d_{0}(0) \rightarrow d(t)$ as a consistent whole should possesses that "ridiculous" property, and any state of any quantum system being invariant to measurement in epistemological considerations as well.

All that can be interpreted that any quantum computer being an intellect recognizing patterns would possesses furthermore "imagination" in the following sense: It figures necessarily a pattern in any data just as a human being can find some sense in any data even in inkblots as those of Rorschach. The difference between "necessarily" and "can" is rather conventional for the free choice of "no pattern" can be accepted as a kind of pattern as the Rorschach tests are often interpreted. However can a quantum computer in a "Chinese room" answer "No pattern!" to some Rorschach test? Yes, it can if it possesses also "self-consciousness" in a sense as in Albert's theorems [1-2], which will be discussed a little above, too.

In fact a restricted series can be monotonically reordered always in two ways, increasing and decreasing, which can have one and the same limit in particular. However the pattern though always recognizable by a quantum computer is also bisected in general. Any quantum computer recognizes two patterns, which can be called complementary in the tradition of quantum mechanics. Indeed if a series is reordered monotonically increasing, this excludes to be reordered monotonically decreasing at the same time in general and vice versa. Even more, an initially converging series as considered 
above allows to be reordered equivalently in another, "complementary" way to another series also converging but to another limit and thus to another "complementary" pattern. One can mock at a quantum computer that it recognizes a pattern really always, but it is also always only the one half of the pattern and no way to be obtained the other and thus the entire pattern to be put together: The utility also in principle of a quantum computer would be zero if this was so.

Even yet the Kochen - Specker theorem [6] as well as John von Neumann's theorem [7] before it elucidated that no "hidden half" of the pattern just as no "hidden variables" in quantum mechanics. However after this is so, where is the other half and how can it be represented explicitly? There is no other option but to be within the first half so that its inaccessibility is due to quantum holism, i.e. an eventual access to it would destroy the first one. Consequently the first half of the recognized pattern is the entire pattern [4]. Of course, that is quite impossible as to any finite pattern thereby to any standard Turing machine, but not if the pattern is infinite, and the Turing machine is quantum. One can see in detail really how the second half of the pattern is "within" the first one:

Any qubit as a unit of quantum pattern consists of two real numbers " $a, b$ ", which are quite independent of each other as long as:

$$
a^{2}+b^{2}<1
$$

There is still a real number "c", but it depends on them as:

$$
a^{2}+b^{2}+c^{2}=1
$$

If any of those three numbers is exactly equal to one, the others are zeros and thereby depend on it. One can interpret those " $a, b$ " as factors correspondingly of discreteness and of continuity or as of a wave and of a particle in the joint quantum motion of anything quantum. If any of those " $a, b$ " is " 1 ", than the case is classical: "either the one, or the other: either discreteness, or continuity; either a particle, or wave". However if not, both components are available.

In the context of pattern recognition, the same means that both "pieces" of calculation and insight are available in a quantum computer just as in an intellect at all. The third number " $c$ " being nonzero corresponds to some nontrivial key for both pieces to be mapped into each other one-to-one and thereby each to be able to decode the other. This key is, first, infinite, and secondly, always different in general: an absolutely unbreakable cipher, so that both pieces are absolutely necessary. Nevertheless a single but infinite choice, which is any qubit as a whole, contents both halves. It can be

anyway considered again as one half only in any quantum system, which includes it.

All this is a way to be interpreted Albert's theorem [1-2]:

If a "quantum automaton" is considered as a whole, it contents the other half within itself and in a sense, it knows its state. For example the missing half can be interpreted as the "halting problem" and thus a quantum computer resolves it as it resolves any problem recognizing a pattern always.

However, changing the viewpoint, moving to outside, beyond the quantum automaton, the entire solving of the problem turns out to be incomplete for the new complete solving should refer to the new quantum system involving the external observer in it as well: A quantum computer resolves necessary any problem, but it is not able to convey the result outside it itself as a physical principle. If a standard computer displays the result to an external observer without changing it, a quantum computer can do it only to an internal observer, i.e. only involving the observation as a part of the task. If one interprets the universe as a quantum computer, which includes our observation as a part of the problem always, it really never "hangs up".

The composed quantum system of a quantum computer and an external observer refers to that only of the standalone quantum computer so: " $a, b$ " can be interpreted as the "radiuses" of the qubits of the computer and observer outside though they are 1 seen inside. Then " $c$ " will correspond to the "radius" of another qubit equivalent to the degree of the entanglement of the systems of the observer and computer. Consequently entanglement can be also interpreted in a computational way 
as the mutual codability of " $a, b$ " corresponding to the "insight" and "continuous advancing calculation" of a quantum computer. The "radius" is proportional to the energy of the corresponding quantum systems since it depends on the ratio between the subspaces " $|0\rangle,|1\rangle$ ” and thus on the frequency “ $\omega$ ".

Summarizing the answer of the second question, the quantum computer turns out to be an intellect in a sense, which can be represented formally and mathematically. Even more, one can raise that whether any intellect has a quantum model, i.e. whether any intellect can be considered as a quantum computer, or whether a "non-quantum intellect” can exist in principle.

Indeed, a quantum computer possesses features seeming obligatory for the definition of intellect such as the universal pattern recognition, "imagination" and "self-consciousness" in a formal sense represented above. However, locked in the "Chinese room", it will be absolutely hopeless because it is not able to convey its answers correctly beyond it. This is not fatal for the intellect test, though, since a human in the same position perhaps cannot answer right being equivalent to a quantum intellect. The discussed model of a quantum Turing machine with qubits for bits implies all those properties.

\section{References}

1. Albert, D.: On quantum-mechanical Automata. Phys. Lett. A 98, 249-252 (1983).

2. Albert, D.: A Quantum-Mechanical Automation. Philos. Sci. 54, 577-585 (1987).

3. Deutsch, D.: Quantum theory, the Church-Turing principle and the universal quantum computer. Proc. R. Soc. London A 400, 97-117 (1985).

4. Deutsch, D.: Quantum Theory as a Universal Physical Theory. Int. J. Theor. Phys. 24, 1-41 (1985).

5. Deutsch, D.: Quantum computational networks. Proc. R. Soc. London A 425, 73-90 (1989).

6. Kochen, S., Specker, E.: The Problem of Hidden Variables in Qunatum Mechanics. J. Math. Mech. 17, 5987 (1968).

7. Neumann, J.: Mathematische Grundlagen der Quantenmechanik. Springer, Berlin (1932).

8. Turing, A.: On computable numbers, with an application to the Entscheidungsproblem. Proc. L. Math. Soc. ser. 242 (1), 230-265 (1937).

9. Yao, A.: Quantum circuit complexity. In: Proceedings of the 34th Annual Symposium on Foundations of Computer Science, pp. 352-361. IEEE Computer Society Press, Los Alamitos, CA (1993). 